\title{
The Use of Dedicated System Tools in Integrated Management in Environmental Protection on the Example of LDAR Program
}

\author{
Arkadiusz Kamiński ${ }^{*}$, Paweł Koziczyński ${ }^{1}$ \\ 1 PKN ORLEN S.A., ul. Chemików 7, 09-411 Płock, Poland \\ * Corresponding author's e-mail: Arkadiusz.Kaminski@orlen.pl
}

\begin{abstract}
Fugitive emissions of volatile organic compounds (VOCs) in the chemical industry are, in addition to channelled emissions, an important element of the total emission into the air. The Leak Detection and Repair (LDAR) program is one of the tools to control this type of emission and is a key component of an integrated approach to environmental management. This paper presents the genesis and general assumptions of LDAR, the methodology of fugitive emissions analysis, including its quantification, an example of how to proceed with the implementation of a comprehensive LDAR system, including tools to support its functioning. The potential environmental and non-environmental benefits of implementing the LDAR program in the refining and petrochemical industry are also presented.
\end{abstract}

Keywords: fugitive emissions, VOC, LDAR, refinery, petrochemistry, integrated environmental management

\section{INTRODUCTION}

Air emission poses a serious problem for the environment. Each activity aimed at processing natural resources involves some discharge of substances into the atmosphere. Taking into consideration a refining and petrochemical plant with its own power supply, one can discern the so-called chanelled emission, i.e. emission of pollutants from every kind of technological and combustion processes, released through stacks, emitters, etc. The sources of this emission include the following: boilers, furnaces, absorber blowouts and other places from which combustion gases (energyrelated pollutants in particular) and waste gases (mainly hydrocarbons), which constitute inherent elements of refining and petrochemical production, are released. It is relatively easy to estimate, quantify and reduce this type of emission. Technologies which curb it are universally accessible and known; they include, among other things, catalytic oxidation, reduction, electrostatic precipitators, adsorbers and absorbers. Fugitive emission, which cannot be omitted when we discuss an integrated approach to environmental management, is slightly different from the chanelled one [Kamiński 2015], and is extremely important in terms of both air protection and optimum operational activity. Only a systemic approach can ensure the expected results of emission reduction.

\section{LDAR PROGRAM}

\section{The origin of the program}

The fugitive emission of volatile organic compounds (VOCs) is significant for the refining and petrochemical industry. According to the Industrial Emission Directive (IED), VOCs are either organic compounds with vapour pressure of at least $0.01 \mathrm{kPa}$ at a temperature of $293.15 \mathrm{~K}$ or organic compounds whose volatility corresponds to this value in special operating conditions.

The obligation to take fugitive emission into account while analysing the impact of installations with integrated permits on atmospheric air quality arises from the provisions of the Environmental Protection Law (articles 144 and 222, paragraph 1). Also, under articles 284 and 285 
of this law, charges for fugitive emission are imposed on those who make use of the environment. Despite a number of legal requirements, the issue of fugitive emission control and reduction has been evaded up to now. Frequently, only the formal requirements were met. In the refining and petrochemical industry, however, the situation changed after the European Commission had published its decision establishing best available techniques (BAT) conclusions for the refining of mineral oil and gas as well as sewage treatment and waste gas purification for the chemical industry. These documents discuss the monitoring and estimation of fugitive VOC emission on the basis of particular methodologies and indicate the need for a comprehensive leak detection and repair system, i.e. the LDAR program. It is a working practice designed for identifying leakage from installation elements; repairing such leaking components can reduce VOC emission. Subject to the requirements of the LDAR program, installation components need to be monitored at regular intervals in order to determine whether they are leaking or tight. Each leaking element has to be repaired or replaced by a set deadline.

In the United States, certain regulations which can be referred to as the beginnings of LDAR were introduced as early as the 1950s. A number of contemporary methodologies originated from the Air Pollution Control Act, which was transformed into the Clean Air Act in 1963, amended in 1970. This Act entitled the American Environmental Protection Agency (EPA) to establish the universal air quality norms protecting the environment and society. LDAR became a formal requirement at the turn of the 20th and 21 st centuries. As a result, the fugitive emission of hydrocarbons had been reduced by $42 \%$ by 2008 as compared with the beginning of the 2000s [LDAR 2017]. However, that was not achieved at no extra cost. According to the refining industry
REF BREF 2015, the annual cost of LDAR for a typical American refinery with over 200,000 fugitive emission points exceeds 750,000 euros.

In Europe, LDAR has been used since the 1990s. In Sweden, it became a compulsory practice for refineries in 1995, which resulted from certain observations made in the late 1980s about the real VOC emission levels being 15-20 times higher than the ones included by reports [Cuclis 2011]. It was not until the 2010s that LDAR was 'noticed' in Poland. It became obligatory for refineries at that time on the strength of the BAT conclusions. Therefore, the requirement to use LDAR as of October 2018 at the latest will have to be included in integrated permits issued for refining installations. Refineries which have not implemented any LDAR system yet will need to do a lot of work consisting in identifying and cataloguing their potential sources of VOC leakage, taking quantitative measurements, formulating emission rates and creating a comprehensive LDAR procedure. It must be stressed here that no details of how to implement the LDAR program are given in the form of legislative acts, so refineries are given considerable latitude in adopting their approach. What matters is merely the ability to show that one's LDAR program works and enables one to achieve the goal of minimising fugitive emission through keeping one's installations in good working order.

Table 1 shows how important the issue of fugitiveemission control is. This table presents statistical data for a typical refinery or a petrochemical plant. Valves and flanged connections are the source of over $90 \%$ of fugitive VOC emission. Research carried out in 17 refineries by the American National Research Council in the late 1990s shows that leaking valves in the refineries could have released into the atmosphere a total of 6,350 tonnes of VOCs per year [Best Practice Guide 2007].

Table 1. Fugitive VOC emission for a typical refinery or petrochemical plant [Best Practice Guide 2007]

\begin{tabular}{|l|c|c|c|c|}
\hline \multicolumn{1}{|c|}{$\begin{array}{c}\text { Elements of the } \\
\text { instalation }\end{array}$} & Quantity (range) & Avarage amount & $\begin{array}{c}\text { Estimated fugitive emission } \\
\text { of VOC [Mg/year] }\end{array}$ & $\begin{array}{c}\text { Participation[\%] } \\
\text { in total emissions }\end{array}$ \\
\hline Pumps & $10-360$ & 100 & 19 & 3 \\
\hline Valves & $150-46000$ & 7400 & 408 & 62 \\
\hline Safety valves & $5-360$ & 90 & 5 & 1 \\
\hline Flange connections & $600-60000$ & 12000 & 201 & 1 \\
\hline Open ends of pips & $1-1600$ & 560 & 9 & 2 \\
\hline Sampling connections & $20-200$ & 80 & 653 & 100 \\
\hline Sum & - & - & & 11 \\
\hline
\end{tabular}




\section{Guidelines}

An innovative, integrated and comprehensive approach to fugitive emission management in a refining and petrochemical plant should be pursued with the use of an LDAR system and computational methods. Monitoring should be done by means of the following:

- photoionisation detectors (PID) and flame ionisation detectors (FID) - the so-called sniffing method connected with correlation curves referring to key installations (quantitative method);

- optical gas imaging - OGI and FLIR cameras (mainly qualitative method);

- index-based computations, periodically verified through measurement.

A complementary, non-obligatory method consists in controlling and quantifying hydrocarbons which occur in the air through periodic campaigns using optical techniques based on absorption, e.g. differential absorption lidar (DIAL) or solar occultation flux (SOF). However, their drawback is that - instead of focusing directly on the source of emission - they mainly show what happens in the air around installations [BREF REF 2015]. Fugitive emission from tanks is determined on the basis of Raoult's law, taking into account vapour liquid equilibrium. Figure 1 shows correlations between qualitative and quantitative methods for determining fugitive emission volume.

The odour detection method and optical gas imaging are inseparable components of each LDAR system because they are used to detect leaks within installations. However, they cannot be used to quantify emissions. A comprehensive approach can be applied through combining odour detection methods with correlation curves in relation to the key elements of installations and the calculation of lengthy emissions. Emission determined in this way becomes a component of the total fugitive emission which constitutes the sum of emission caused by leakage and emission occurring during normal installation operation [Kanderska 2015].

Subject to the requirements of the LDAR program, installation components need to be monitored at regular intervals in order to determine whether their elements are leaking or tight. Each leaking element has to be repaired or replaced by a set deadline. According to the data included in Best Practice Guide 2007, the implementation of an LDAR system enables one to eliminate around $63 \%$ of VOC emission coming from refinery installation leakage and around $56 \%$ of VOC emission coming from the production of organic chemicals.

When properly run, the LDAR program comes in the following five steps [Best Practice Guide 2007]:

1. Identification of elements - ascribing explicit identifiers to potentially leaking elements on the basis of, for instance, P\&ID diagrams and databases including information on location, medium, emission index, last repair, etc.;

2. Leakage definition - one may follow the example of the American 'Method 21' [US EPA 2017], which defines a leakage as untightness resulting in emission exceeding 10,000 ppm. One may assume other threshold values or observe the visual changes of a given element.

3. Monitoring of leakage detection and VOC emission by means of portable devices and methodologies described in BAT 6 (see Table 1). The monitoring frequency of 2-8 years suggested by US EPA in Method 21 depends on the type of component and periodical rates of its flight.

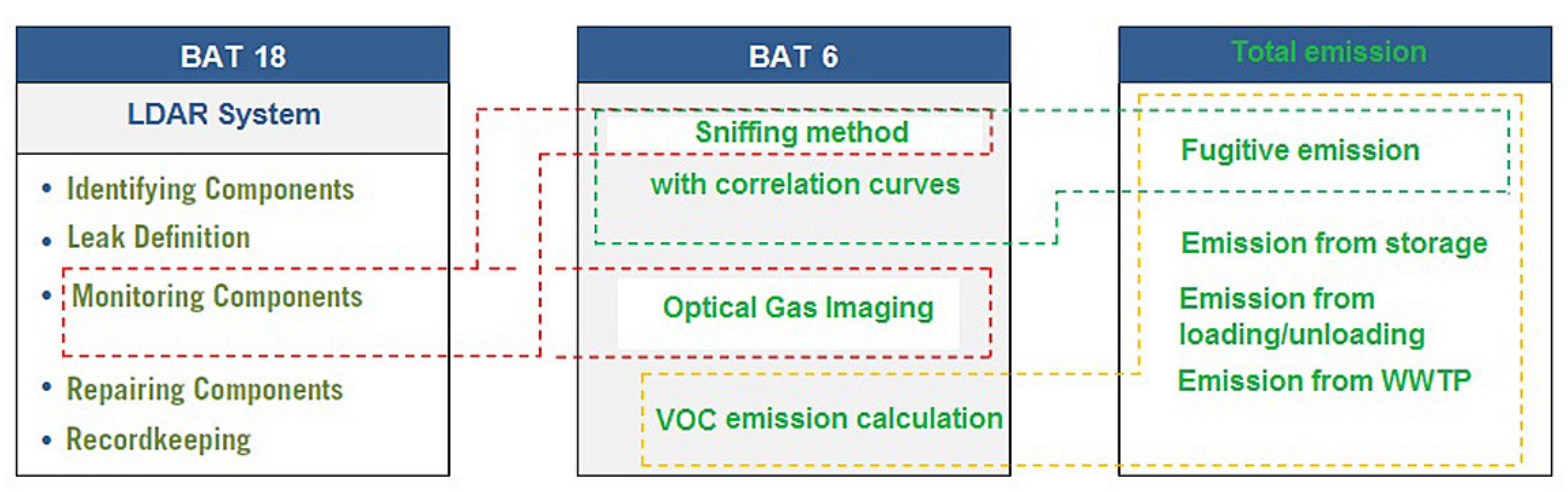

Fig. 1. Correlations between qualitative and quantitative methods for determining fugitive VOC emissions [Kanderska 2015] 
4. Repair - in certain cases it may be sufficient to tighten bolts, replace bolts, tighten gland nuts and inject lubricant. Some elements may be listed for later repair (the exact date should be included in the documentation). Elements are considered repaired when the very next tightness test shows that the leakage threshold has not been exceeded.

5. Recordkeeping - all the elements which may be leaking must be listed; equipment specifications (description of changes) as well as tightness monitoring results must be stored; additionally, each repair and inspection test need to be recorded.

A significant advantage brought indirectly by the LDAR program is the chance to quantify the fugitive VOC emission on the basis of the survey made in step no. 3 according to EN 15446:2008 standard or its amended version. The fixed emission indexes and patterns of correlation curves presented in Table 2 may be used interchangeably, but the amended version of the standard is preferred in official legal documents and norms (Method 21 US EPA 2017) as well as specialist literature [Best Practice Guide 2007, Gonzalez et al. 2015].

\section{Practical utilisation of the LDAR program}

It must be mentioned here that despite the lack of legal obligation to implement the LDAR program, refineries have been inspecting their potentially leaking components as well as sealing and repairing their installations. As a result, they now need to systematise their current solutions and procedures, organise them into an integrated system combining BAT 6 and BAT 18, and assign certain roles to particular organisational sections. The way in which a refinery implements the LDAR program largely depends on its past experiences, complexity and certain local conditioning, including public expectations.

Preparatory works should consist in dividing the plant into separate installations (this division is most often consistent with the division in the integrated permit) and then each installation into process streams. Since in most cases the production and/or technology departments have the most detailed knowledge of these divisions, it is them that are most actively involved in the preparatory works. The very next step is to establish the number and types of fugitive emission points for each process line. Points should

Table 2. VOC emission factors [Gonzalez et al. 2015]

\begin{tabular}{|c|c|c|c|}
\hline \multicolumn{4}{|c|}{ Fixed emission factors } \\
\hline Elements of the instalation & Medium & $\begin{array}{l}\text { Coefficient[kg/h/element] for } \\
\text { emissions } \geq 10000 \text { ppm }\end{array}$ & $\begin{array}{l}\text { Coefficient[kg/h/element] for } \\
\text { emissions }<10000 \text { ppm }\end{array}$ \\
\hline \multirow{2}{*}{ Pumps } & Light liquid & 0.437 & 0.012 \\
\hline & Heavy liquid & 0.3885 & 0.0135 \\
\hline \multirow{3}{*}{ Valves } & Gas & 0.2626 & 0.0006 \\
\hline & Light liqiud & 0.0852 & 0.0017 \\
\hline & Heavy liquid & 0.00023 & 0.00023 \\
\hline Safety valves & gas & 1.691 & 0.0477 \\
\hline Flange connections & All & 0.0375 & 0.00006 \\
\hline Open ends of pipe & All & 0.01195 & 0.0015 \\
\hline Compressors & Gas & 1.608 & 0.0894 \\
\hline \multicolumn{4}{|c|}{ Correlation curves } \\
\hline \multicolumn{2}{|c|}{ Emission SV $\geq 100000$ ppm } & \multicolumn{2}{|c|}{ Emission SV < 100000 ppm } \\
\hline \multicolumn{2}{|c|}{ coefficient $[\mathrm{kg} / \mathrm{h} /$ element $]=\mathrm{C}$} & \multicolumn{2}{|c|}{ coefficient $[\mathrm{kg} / \mathrm{h} /$ element $]=\mathrm{A} \times(\mathrm{SV})^{\mathrm{B}}$} \\
\hline Elements of instalation & Constant value $\mathrm{C}[\mathrm{kg} / \mathrm{h} / \mathrm{element}]$ & Coefficient A & Coefficient B \\
\hline Pumps & 0.16 & 0.0000503 & 0.610 \\
\hline Valves & 0.14 & 0.00000229 & 0.746 \\
\hline Flanges & 0.084 & 0.00000461 & 0.703 \\
\hline Other flanges & 0.03 & 0.00000153 & 0.735 \\
\hline open ends of pipe & 0.079 & 0.0000022 & 0.704 \\
\hline Other & 0.11 & 0.0000136 & 0.589 \\
\hline
\end{tabular}


be understood as details, including the smallest ones, which may be sources of emission. A given set of points forms an element which is illustrated in piping and instrumentation diagrams (P\&ID). Thus, for instance, a valve is an element with points in the form of three flanged connections and a gland. A flanged connection at a pipeline is an example of a one-point element. The elements which should be inventoried include, among other things, the following: mixers, pumps, compressors, flanges, open pipe ends, valves, sample collection points. Figure 2 presents an example of how to mark process streams and elements which are subject to LDAR.

Since the LDAR program is connected with preventing VOC emission, it is necessary to determine the threshold value for volatile hydrocarbons in the process streams; when the value is exceeded, the volatile hydrocarbons will be classified as those which are subject to LDAR. The purpose is to avoid inventorying water streams or those with inorganic substances. The threshold value depends on a number of factors and is not legally imposed; it is selected individually.

An important component of the LDAR system is the value of leakage threshold, i.e. the level of VOC emission expressed in ppm. When untightness exceeds this threshold, it is classified as leakage and is subject to special repair procedures. The value of this threshold is selected individually, too.

In order to use the data collected in the process of inventorying for quantifying the fugitive VOC emission, among other things, one has to determine the composition of process streams. Knowing this composition, we can convert the emission of total organic carbon (expressed in ppm) measured by means of a PID detector into the emission of a particular substance expressed in $\mathrm{kg} / \mathrm{h}$, additionally using the correlations presented in

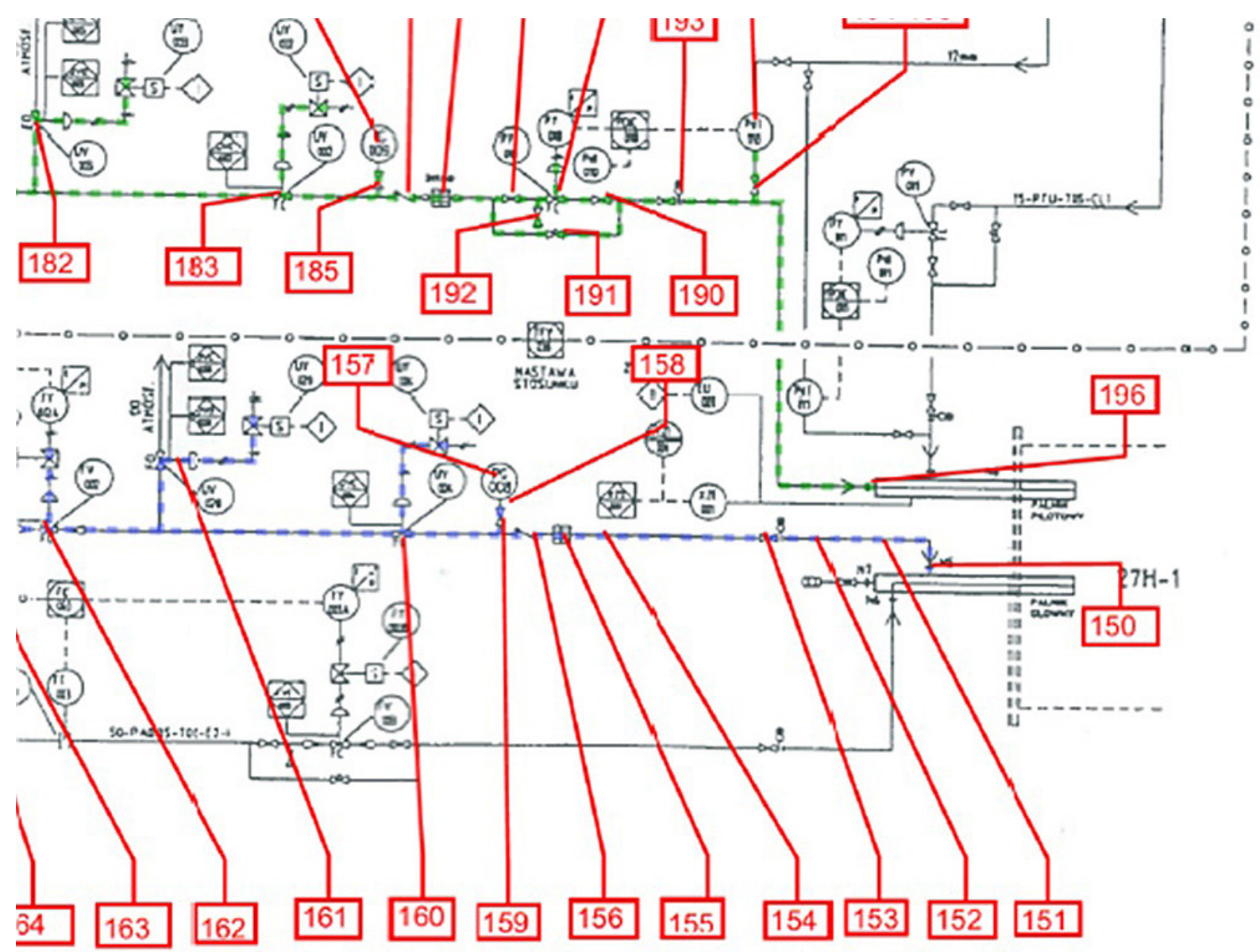

\section{Stream 9 - low pressure fuel gas for pilots}

\section{Stream 10 - low pressure fuel gas for burners}

Fig. 2. An example of process streams establishing and instrumentation marking, based on [Kanderska 2015] 
Table 2. To simplify this conversion, one can assume that the percentage composition of a liquid stream corresponds to the composition of vapours.

One can carry out the field inventorying by engaging one's own production and maintenance specialists or outsourcing this service. It is crucial to make sure that the people responsible for the inventorying are experienced, preferably in terms of a given installation, which shortens the time needed for inventorying and enables the people to avoid making mistakes. Inventorying one's elements, one needs to designate each of them. If there already exist some symbols in one's plant, there are obviously no obstacles to using them. It must be taken into account that some elements may be inaccessible (no access; areas requiring the use of oxygen respirators, etc). Such elements can be inventoried when they become accessible.

One way to designate elements in the field is to affix designation labels to them. Such labels have to be made of durable materials resistant to weather and the environment of the plant (hydrocarbon vapours, acids, etc). These labels have to be affixed in such a way that they do not hinder the everyday usage of installations and, at the same time, are not accidentally dismantled; this is particularly important when it comes to installation repairs during which the labels may be lost or wrongly re-affixed.

Measurements and leakage repairs are the most important components of the LDAR program. Alike inventorying, they can be carried out by one's own specialists or outsourced. In large plants where the number of points is over 300,000 such measurements and repairs are outsourced. The frequency and scope of monitoring campaigns should be selected individually. Experience [BREF REF 2015, Best Practice Guide 2007] shows that the frequency may range from once every three or four years to several times a year. It seems to be rational to organise such monitoring campaigns at least as often as the repairs.

The measurements are performed first by means of OGI optical cameras and then, if leakage is detected in the form of a cloud, flame ionisation detectors are used. When leakage is identified (i.e. untightness exceeds the assumed threshold) through planned (routine) or extraordinary measurements, it needs to be marked by means of a designation label, for instance, and reported as earmarked for the first repair attempt. Maintenance specialists usually verify whether it is possible to repair a leakage or not. The procedure of the first repair attempt is adopted when the repair is approved. In large industrial complexes, such repairs usually consist in tightening bolts only. As a general rule, no extensive repairs are possible due to guarantee restrictions, regulations imposed by technical inspection offices and the risk of further damage to the element. It must also be remembered that even tightening a bolt may be regarded as a repair process and may need to be agreed on internally. After a repair has been completed, its effectiveness is evaluated through a control measurement. Control teams and repair teams may work separately or together. One of the good practices is to carry out measurements during the repairs.

If the repair of a given point is not approved or ineffective, the point is earmarked for proper repair usually done when the installation stoppage. Stopping an installation because of a leak only is rational for neither environmental nor economic reasons (flare stack dumps and production losses respectively). The measurement confirming the effectiveness of a repair is done after the installation has been restarted; the medium needs to be present in the stream.

Both first and proper leakage repairs should take place as early as possible; however, there are no deadlines imposed by LDAR regulations. Deadlines should be clearly set depending on individual factors and included in plants' internal procedures.

The effective and integrated management of the LDAR program may be facilitated by a dedicated software application. One may choose from a wide range of ready-made databases or customised applications. The main purpose of using such applications is to support particular organisational units in terms of inspecting their production installations for the identification of fugitive emission, repair and reporting. The basis of such applications is formed by an extended database including information on elements, points and process streams as well as photographs of elements with their labels. Such properly prepared software also helps to plan measurements, issue repair notices and calculate emission volumes. The LDAR system and its software may be administered by environmental protection or maintenance departments. In either case, the people responsible for such administering should be assigned to this particular area only due to its scope; they need to cooperate closely with other specialists involved. 


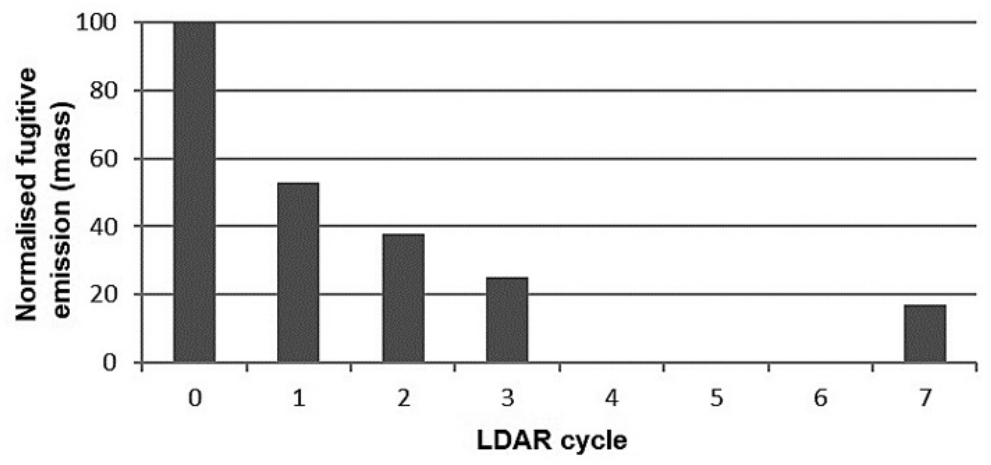

Fig. 3. Relative VOC emission for one of the European refineries, assuming that the emission before the implementation of LDAR was at the level of 100\% [Gonzalez et al. 2015].

\section{CONCLUSIONS}

An example of the integrated approach to environmental management, LDAR benefits the environment as it lowers VOC emissions into the atmosphere. When properly run by maintenance crews and technological specialists responsible for production installations, LDAR reduces fuel, medium and product losses, improves workplace safety and generates savings in terms of environmental fees. Data collected by CONCAWE [Gonzalez et al. 2015] include results produced by one of the European refineries over several LDAR cycles. Figure 3 shows that it is possible to decrease mass fugitive VOC emission fivefold over a longer period of time. The study [Drago 2010] indicates that between 1988 and 2008 emission reductions in the United States reached $79 \%$ and $82 \%$ in the case of refinery installations and chemical industry installations.

It takes several or even a dozen or so years to produce notable effects of LDAR. We will be able to share our Polish LDAR experiences in a dozen or so years because LDAR has been implemented in Polish refineries since October 2018. Nevertheless, it can already be seen that there are and will be some challenges which must be confronted. It must also be remembered that the approach presented in this article is merely an example of how LDAR can be run; the approach is not imposed by law or obligatory in any way.

\section{REFERENCES}

1. A Simplified Timeline of the History of LDAR (Leak Detection and Repair) http://www.targetemission. com/2017/12/27/simplified-timeline-history-ldarleak-detection-repair/ dostęp 20-07-2018;

2. Cuclis A., 2011. Measuring VOCs in Refineries and Chemical Plants, Houston Advanced Research Center, Houston;

3. Drago J., 2010. Enhanced Leak Detection and Repair Programs for the Chemical Processing Industry, materiały konferencyjne ChemInnovations, Houston;

4. Gonzalez L. et al. Techniques for detecting and quantifying fugitive emissions - results on comparative field studies, CONCAWE, Brussels, 2015;

5. Kamiński A., Koziczyński P. 2015. Zintegrowane podejście do zagadnień ochrony środowiska $\mathrm{w}$ kompleksie rafineryjno-petrochemiczno-energetycznym w obliczu środowiskowych regulacji prawnych. Chemik, 69, 10, 635-638;

6. Kanderska K., 2015. Documentation prepared by ORLEN Eko Sp. z o.o. as an order by PKN ORLEN S.A., December;

7. REF BREF, European Commission: Best Available Techniques (BAT) Reference Document for the Refining of Mineral Oil and Gas, March 2015;

8. Best Practices Guide, Leak Detection and Repair; US EPA, Washington, 2007;

9. Method 21 - Determination of Volatile Organic Compound Leaks, US EPA, https://www.epa.gov/ sites/production/files/2017-08/documents/method_21.pdf; available 20-07-2018. 